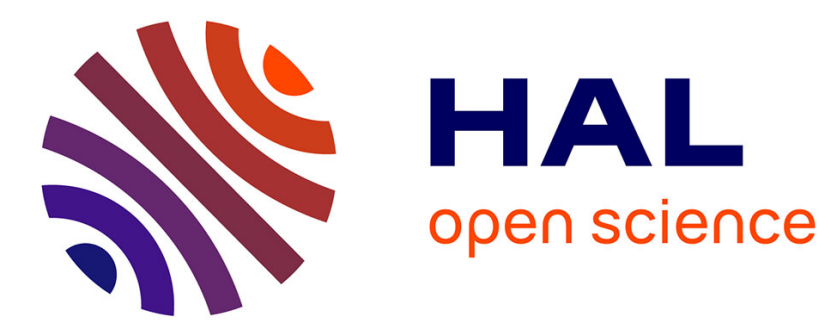

\title{
En quête des raisons de l'autre.
}

Christine Bouissou

\section{To cite this version:}

Christine Bouissou. En quête des raisons de l'autre.. Le Télémaque. Philosophie, Education, Société, 2015. halshs-00834917v2

\section{HAL Id: halshs-00834917 https://shs.hal.science/halshs-00834917v2}

Submitted on 14 Jan 2016

HAL is a multi-disciplinary open access archive for the deposit and dissemination of scientific research documents, whether they are published or not. The documents may come from teaching and research institutions in France or abroad, or from public or private research centers.
L'archive ouverte pluridisciplinaire HAL, est destinée au dépôt et à la diffusion de documents scientifiques de niveau recherche, publiés ou non, émanant des établissements d'enseignement et de recherche français ou étrangers, des laboratoires publics ou privés. 


\title{
Christine Bouissou Christine.bouissou@univ-paris8.fr
}

\author{
Université Paris 8 Vincennes Saint-Denis
}

Téléphone : 0663854772

\section{Titre : En quête des raisons de l'autre}

(Autre titre possible: ( $\left.S^{\prime}\right)$ éduquer : retrouver des mobiles et des voix d'enfants)

Mots clés : enfance, émancipation, culture, écoute, transmission

\section{Résumé}

Ce texte est une réflexion sur les questions d'émancipation vis-à-vis des ancrages normatifs et d'altération des espaces symboliques. L'étude porte sur un reportage radiophonique au cours duquel des jeunes filles, vivant dans une banlieue francilienne, se présentent et mettent leur vie en récit. Si l'on saisit d'abord seulement des considérations anecdotiques, une autre écoute est possible. Etayée notamment par les travaux philosophiques sur le rapport à l'enfance dans le monde contemporain et par l'approche psychanalytique et littéraire de J. Kristeva notamment, l'analyse permet d'avancer quelques pistes concernant la manière dont on peut comprendre et aider à l'émancipation, par le travail psychique de questionnement, de mise en crise des standards sémantiques dont les sujets - ici des femmes - s'autorisent. La mobilité, la plasticité psychique sont des signes de solidité interne, permettant une normativité non crispée, mais en travail, une raison non dogmatique, mais inscrite dans le collectif et intégrative de l'altérité/adversité. 


\title{
Titre : En quête des raisons de l'autre
}

\begin{abstract}
«Je nous vois, nous, hommes de ce siècle, avoir ce privilège, ce regard sur les traditions mondiales qui nous nourrissent profondément, qui sont matière de réunification pour être dans le siècle en maintenant l'entretien avec les morts, l'entretien avec les anciens. Je crois beaucoup à la fois à l'expérience de l'écriture, de la réflexion, qui est une aventure tout à fait ouverte dans le tâtonnement, dans le risque, dans la confrontation avec l'in-ouï, dans la manière de vouloir grignoter vers des parcelles de l'inexploré mais en contact perpétuel avec le fond, avec le mémoriel, avec l'ancien, lorsque cet ancien peut encore nous parler, peut avoir cette capacité d'appartenir à notre actuel, de quelque langue qu'il vienne, de quelque siècle qu'il perdure, jusqu'à parvenir à nos oreilles. »

Abdelwahab Meddeb, For intérieur, France Culture, 10 juin 2011
\end{abstract}

\section{Introduction}

Le philosophe italien G. Agamben ${ }^{1}$ a alerté sur l'évacuation très problématique de l'imagination dans le monde européen contemporain de la constitution des savoirs. La montée en puissance de l'expérimentation et l'opposition entre ordres objectif et subjectif évacuent à tort du monde de la connaissance, le sensible et l'imaginaire. La coupure assèche les vies psychiques et stérilise les transmissions. Si l'on veut produire autre chose, il faut faire circuler les divers types de connaissances, faire se rencontrer leurs modes d'élaboration, favoriser les hybridations.

\section{Enfance et histoire}

Agamben propose une réflexion sur l'expérience et sa mise en mots, dans son ouvrage «Enfance et histoire. Essai sur la destruction de l'expérience ». Inspiré des thèses de W. Benjamin $^{2}$, il analyse ce processus de disparition de l'expérience ${ }^{3}$ qui s'est produit au cours du $20^{\text {ème }}$ siècle dans les sociétés européennes. Il s'explique par une accélération considérable des rythmes de vie, des progrès techniques, des changements sociaux. Densification, complexification produisent une multiplication des sens et une perte d'orientation. L'autre face du trop-plein de sens est le sentiment de vide intérieur, de banalité du monde et de soi qui nous guette tout autant. Plusieurs penseurs ont convergé - W. Benjamin ${ }^{4}$, S. Weil ${ }^{5}, \mathrm{H}$. Arendt $^{6}$, notamment -, épouvantés par les catastrophes humaines dont ils étaient contemporains, cherchant à organiser par leur raison, les processus qui y avaient conduit et leurs conséquences pour les générations à venir. L'ordinarité du quotidien qui constituait jadis la matière première de l'expérience et que chaque génération transmettait à la suivante a disparu et conduit aux problèmes contemporains de transmission intergénérationnelle : les

\footnotetext{
${ }^{1}$ Agamben, G. 1989. Enfance et histoire, Paris, Petite Bibliothèque Payot (1978 $1^{\text {ère }}$ éd.).

${ }^{2}$ Benjamin, W. 1989. Paris, capitale du $19^{\text {ème }}$ siècle, Paris, Ed. Cerf (1939 $1^{\text {ère }}$ éd.).

${ }^{3}$ Les termes de destruction ou de disparition de l'expérience ont un caractère apocalyptique auquel nous pourrions préférer l'idée de crise, de tension, de risque d'éclatement mettant en péril les identités et leur intégrité telle que la décrit Didi-Huberman (Didi-Huberman, G. 2009. Survivance des lucioles, Paris, Ed. Minuit).

${ }^{4}$ Benjamin, W. 2000. Euvres III, Sur le concept d'histoire, Paris, Folio Gallimard (1940 $1{ }^{\text {ère }}$ éd.).

${ }^{5}$ Weil, S. 1949. L'enracinement, Paris, Gallimard.

${ }^{6}$ Arendt, H. 1972. La brèche entre le passé et le futur, in La crise de l'éducation, Paris, Gallimard (1954 1 1̀ेe éd.).
} 
aînés seraient dans la difficultés voire l'impossibilité de transmettre aux plus jeunes ce qu'ils ont vécu, connu, voire ils ne s'y autoriseraient plus car «l'expérience trouve son nécessaire corrélat, moins dans la connaissance que dans l'autorité, c'est-à-dire dans la parole et le récit $»^{7}$. Il observe corrélativement le refus de l'expérience de certains jeunes, conséquence de l'impuissance des aînés à leur livrer, leur transmettre, une expérience " digne de ce nom », c'est-à-dire fondée sur des événements et des épreuves à la fois réels et suffisamment élaborés. «Sa condition a beau être objectivement terrible, on n'a jamais vu spectacle plus répugnant que celui d'une génération d'adultes qui, après avoir détruit toute possibilité d'expérience authentique, impute sa propre misère à une jeunesse désormais incapable d'expérimenter. Quand à une humanité effectivement dépossédée de son expérience on prétend imposer, comme aux rats du labyrinthe, une expérience manipulée et directive, autrement dit quand il n'y a d'expérience possible que dans l'horreur ou le mensonge, alors le refus de l'expérience peut constituer - provisoirement - une défense légitime $»^{8}$. L'affaiblissement de l'expérience en tant que conséquence de l'accélération de la marche des sociétés occidentales sur les plans technique et politique est aussi corrélatif de la coupure entre le monde de la science et l'espace du désir, de l'imagination, du fantasme. La science moderne est née d'une méfiance envers l'expérience telle que la concevait la tradition et pour laquelle « elle ne relevait pas du subjectif mais elle se définissait plutôt comme la coïncidence du subjectif et de l'objectif, de l'intérieur et de l'extérieur, du sensible et de l'intelligible » ${ }^{9}$. L'expérience relève en effet d'un processus de métabolisation des événements vécus, grâce à l'imagination laquelle a constitué pour les Anciens, non pas un obstacle mais plutôt le medium par excellence de la connaissance. De ce point de vue, les savoirs acquis en état de veille restent inefficaces s'ils ne sont pas rêvés pour acquérir une puissance active : sans imagination, pas de compréhension possible pour l'homme. Agamben réactualise une piste de travail : redonner une place à l'imagination et au désir ; permettre de métaboliser et d'élaborer les événements vécus, faire communiquer le monde du sensible et le monde de l'intelligible. Car la fertilité de la connaissance, sa créativité et son inventivité nécessitent pour advenir, d'être imaginées à partir du réel.

Agamben formule dans le même essai une autre proposition vivifiante : expérience et langage se prolongent et renvoient circulairement l'un à l'autre. Dans l'enfance prend naissance le langage, qui va la constituer. Elle n'est pas le paradis que nous quitterions définitivement un jour pour nous mettre à parler ; il faut renoncer à chercher son origine et à la définir comme un point dans une chronologie, comme une cause initiale séparant dans le temps un avant-soi d'un après-soi. L'enfance devient ce que l'on peut dire, par l'appropriation d'une langue d'abord étrangère, bien que maternelle. C'est dans ce cercle précisément qu'il faut chercher le lieu de l'expérience en tant qu'enfance de l'homme.

\section{Enfance et parole}

La figure de l'enfant revêt une fonction primordiale : elle est celle que l'on piste, que l'on cherche à faire surgir, par un retour sur des textes, des données de recherche, des problématiques posées à propos de l'acte éducatif. L'enfant est une voix à exprimer et à accueillir, hors d'âge parce que de tous les âges ; l'enfance est devant soi, elle s'élabore, existe, sort d'elle-même. Son temps n'est pas figé, clos, arrêté derrière soi. Parler, trouver ses mots et sa langue, entrer dans le sémantique font émerger des limbes du sémiotique. L'ordre du temps peut être renversé et sans doute aussi les ordres normatifs et générationnels.

\footnotetext{
${ }^{7}$ Agamben, G. 1989. Enfance et histoire, Paris, Petite Bibliothèque Payot (1978 $1^{\text {ère }}$ éd.), p. 26.

${ }^{8}$ Ibid., p. 29.

${ }^{9}$ Ibid., p. 46-47.
} 
Nous tentons de penser conjointement l'enfance, l'histoire et un troisième terme, l'exil, en prenant appui sur le philosophe $\mathrm{J}$. Borrell ${ }^{10}$ pour qui le pays natal condamne à l'isolement car « il n'y a de naissance qu'en terre étrangère ». Grandir suppose de partir, de se départir du sol, de pouvoir en parler depuis l'ailleurs, de s'enraciner dans une raison autre. Celui qui parle est d'abord interprète, traducteur, go-between. La perspective engage par conséquent à chercher à identifier des signes de mutation et différence/différance, plus que d'évolution ou de progrès. L'enfant n'est pas seulement 'le fils ou la fille de...', il est l'enfant que chacun porte en luimême, celui qu'il a été et qu'il devient. Parce que tout adulte en souffrance est un enfant qui cherche à parler, il est possible de l'aider à retrouver contact, en (se) mettant en mots. La parole délivre et devance, elle est un voyage de la chair hors du corps humain par la voix, un exit, un exil, un exode. Elle attend et accueille. "Ce que tu ne sais pas, dis-le. Ce que tu ne possèdes pas, donne-le. Ce dont on ne peut parler, c'est cela qu'il faut dire ». Ces réflexions sont celles de Valère Novarina ${ }^{11}$ à propos de son travail théâtral qu'il conçoit comme un acte de création de soi, cherchant à promouvoir des déplacements et des transformations. La parole nous a été donnée non pour parler mais pour entendre ce qui est tu.

Les occasions de chercher à entendre, au sens d'ouïr et de comprendre, foisonnent et les entretiens de recherche, thérapeutiques, journalistiques sont potentiellement des techniques de l'éveil. Le 'faire parler' est un art, une tekhnè, un mode d'existence et un rapport à l'autre ; la reformulation l'une de ses richesses : reprise, partage et confirmation des mots de chacun. L'entretien est aussi un acte professionnel pour un chercheur, un journaliste, un interrogateur des questions humaines. Qu'est-ce qu'on relève de la parole de l'autre ? Qu'est-ce qui fait événement, mérite d'être entendu ? Cet ethos de la rencontre suscite une autre question : Comment soutenir la vulnérabilité, «en tant que doublure inséparable de la fraternité ${ }^{12}$ ? Peut-être en la cultivant.

\section{En quête...}

Nous présentons dans les lignes qui suivent un travail d'analyse, de recherche d'interprétation, mené à partir d'un reportage radiophonique diffusé sur les ondes de la radio nationale publique France Culture ${ }^{13}$. Inscrit dans la pensée du monde contemporain, ce média propose des programmes conçus autour de reportages et d'interviews, qui sont autant d'espaces de prise de parole publique et ordinaire, de mise en mots des expériences et d'appui à la constitution de celles-ci, de part et d'autre du poste de radio. Dans le cas présent, un journaliste entouré d'un groupe de jeunes filles les questionne sur leur vie dans une ville de banlieue francilienne, Champigny sur Marne. Leurs parents ont émigré en France depuis des pays bordant la Méditerranée. Une heure de reportage permet à l'auditeur d'entendre ces filles parler d'elles-mêmes, des parents, de leurs pairs garçons et des cadres dans lesquels s'inscrit leur existence, leurs limites et leurs possibles. Elles décrivent les rapports spatiaux, familiaux, amicaux, et leur lot de contraintes: ' Je dis pas forcément que je les respecte mais elles sont là '. On comprend qu'elles s'y adossent, s'en saisissent et en sortent. Elaborant collectivement un récit, elles incitent l'auditeur à une élaboration de questions actuelles et sensibles : la vie dans les banlieues, les rapports hommes-femmes, l'identité française, l'immigration, les rapports Nord-Sud, le passé colonial français et la figure du garçon arabe. Sujets brûlants, à rafraîchir.

Un arrière-plan d'interrogations a orienté notre écoute - les écoutes devrait-on dire, stimulées par des lectures et en stimulant d'autres en retour - le tout en rapport avec les questions que

\footnotetext{
${ }^{10}$ Borrell, J. 1996. La raison de l'autre, Paris, L'Harmattan, p. 43.

${ }^{11}$ Novarina, V. 1999. Devant la parole, Paris, Pol, p.29.

${ }^{12}$ Kristeva, J. 2005. La haine et le pardon, Paris, Fayard, p. 120.

${ }^{13}$ 'Ma cité mon cocon. Jeunes filles entre elles et entre soi', France Culture, Emission Terrains sensibles, 2009.
} 
nous travaillons en tant que chercheur inscrit dans une époque et un territoire particuliers l'université de Saint-Denis, au nord de Paris - creusant des questions autour de l'élaboration, du déplacement et de l'altération des frontières disciplinaires et des espaces symboliques.

Un reportage est un programme destiné à informer les récepteurs sur un événement d'actualité, sur une activité humaine. Aller interroger quelques filles habitant une ville de banlieue c'est faire de ce qu'elles vivent et peuvent en dire, un fait d'actualité qui mérite d'être diffusé sur des ondes nationales (et si ça le mérite, ça se mérite aussi). Il s'agit de relever une chose d'abord ordinaire, banale, pour la hisser au rang d'événement. Ici tous participent de cette opération: l'interviewer, les filles, l'auditeur. Au bout des ondes, ce dernier prête attention à ce qu'il entend, tente d'en comprendre quelque chose : à quel jeu se livrent les personnes en présence, qui questionne qui, quelle est l'histoire... Autant de questions mises au travail, par la rencontre d'un espace public polyphonique et de l'espace privé.

Nous avons fait l'hypothèse que les propos des jeunes filles interviewées suscitent un retour vers l'enfance, la leur, celle de leurs frères, celle de leurs parents, et aussi la nôtre, auditeurs. Si l'étude que nous présentons a commencé dès l'instant de sa diffusion, elle a pris ensuite un relief particulier, un sens plus précis, une allure plus vive, en raison des actualités moyenorientales et occidentales et leurs résonnances sur la façon dont est pensée en France l'intégration des populations issues de l'immigration. Dans le prolongement de nos propres travaux sur le développement et la trajectoire des enfants et adolescents, filles et garçons, nous avons souhaité élargir la focale en portant notre intérêt sur des phénomènes moins connus et moins accessibles, tels que la transmission père-fille, les conditions de prise de parole en tant qu'acte civique de responsabilité et de reconnaissance, et les manières de promouvoir, par l'écoute et le dialogue, l'altération des systèmes symboliques. Dans ce cadre, certaines émissions diffusées sur France Culture telles que For intérieur ou Cultures d'islam constituent un corpus et se prêtent à l'analyse. Les invités se succèdent, exposent leur travail de recherche et de mise en correspondance des espaces géographiques et symboliques - le Nord et le Sud de la méditerranée, le Coran, la Bible et des textes littéraires, philosophiques et proposent des pistes originales de réflexion.

«Et si la méditerranée n'était qu'un fossé fantasmé ou mieux encore, un pont entre les deux rives de la méditerranée ?» proposait l'écrivain, poète érudit et animateur de radio A. Meddeb. Dans son œuvre il s'attachait à opérer un tel rapprochement par le travail de la pensée, de la recherche et de l'écriture, et à trouver un sol commun profond entre ce qui a pu s'exprimer en langue arabe, en langue persane ou en langue latine et dans l'émergence des vulgates européennes. "Il est temps que cette séparation qui nous semble arbitraire entre la rive nord de la méditerranée et sa rive sud, tombe enfin ${ }^{14}$. L'auteur observe que par la circulation, les frontières parfois s'estompent (des actions politiques permettent d'avancer dans ce sens). Mais il y a des structures plus profondes qui n'ont pas encore émergé et que chacun porte en lui. A. Meddeb nous enjoint de jouer et de faire en sorte que ce qui se reçoit à la superficie des choses puisse retrouver dans les profondeurs, les raisons mêmes du dépassement des identités particulières et de refondation de l'identité de l'homme à partir d'elles, en poésie comme en politique ${ }^{15}$. Les voies de cette refondation - dont l'écriture est un véhicule - traversent des espaces symboliques multiples, les réveillent, les créolisent, comme l'appelait de ses vœux E. Glissant ${ }^{16}$. Essais, récits, poésie participent de l'ouverture.

\footnotetext{
${ }^{14}$ Meddeb, A. For intérieur, France Culture, 10 juin 2011.

${ }^{15}$ Meddeb, A. 2011. Printemps de Tunis, la métamorphose de l'histoire, Paris, A Michel.

${ }^{16}$ Glissant, E. 1990. Poétique de la relation, Paris, Gallimard.
} 
A. Meddeb veut ouvrir les vulgates européennes. Quelles sont celles qui concernent les femmes originaires des pays du Sud vivant dans les banlieues?

Ces femmes seraient doublement, triplement assujetties : par leur situation économique, leur culture et leur sexe. C'est en ces termes que souvent l'on parle d'elles, réprouvant leur isolement dans les ghettos - cités mises au ban et corps voilés. Mais c'est consentir aux forces d'assignation et faire peu de cas de l'énergie qui circule intra et inter-subjectivement. Comment travailler, afin de comprendre les mécanismes d'assignation et les voies d'émancipation et s'attacher à favoriser ces dernières ? Un premier élément de réponse consiste à revenir à la pratique de l'interprétation et à la cultiver avec un soin très particulier, comme le soutient Y. Citton en vue de l'émergence d'une nouvelle économie de la connaissance $^{17}$ : la dynamique propre à l'interprétation est faite de tâtonnements - où l'intuition (esthétique) joue un rôle aussi important que la systématicité (scientifique). C'est sur ce point que peuvent porter les efforts tant du pédagogue que du chercheur : chercher, en prenant appui sur les textes philosophiques et psychanalytiques, une invention pédagogique et une opérativité de la pensée pour promouvoir l'émancipation, le dépassement de soi et le droit de cité.

Notre travail s'appuie en outre sur le fait que l'univocité des effets d'assignation n'est pas démontrée - comme les travaux sur l'intersectionnalité s'attachent à l'étudier ${ }^{18}$. Au contraire, il y a fort à parier que les champs de forces - être une femme, vivre en banlieue, être issue de pays du Sud/ex-colonisés - entrent en jeu, en résonance et plus qu'ils ne se renforcent, souvent se défont. Le passé colonial français, négligé, le rabaissement de la culture arabe sous le voile de la religion musulmane considérée dans ses plus grands excès, sa richesse insoupçonnée, la pesanteur des dictats sociaux qui pèsent sur les hommes aussi, ont été longuement occultés par les vulgates mais font partie des savoirs et des ressources de certain(e)s qui peuvent y prendre appui pour esquiver les effets d'assignation et devenir autres.

\section{... des raisons de l'autre}

Le reportage radiophonique «Ma cité mon cocon » constituant notre corpus invite à chercher des possibles plus que des limites et à former ou réformer l'oreille dans ce sens. Sont au travail les questions de réception, d'hospitalité, de travail et de chemin de l'auditeur, parallèle à celui des filles interviewées. Celui que l'on cherche à transformer, c'est d'abord soi-même. Notre projet a débuté par un exercice d'écriture littéraire ${ }^{19}$, exercice littéraire permettant une relance du travail d'élaboration, guidé par la pensée de G. Deleuze et F. Guattari ${ }^{20}$ et l'intérêt pour les processus minoritaires, voies d'échappées des lieux communs, postulant que sortir de chez soi, de l'immeuble et de la cité aide à s'émanciper des ancrages normatifs : non pas voie en sens unique mais double déterritorialisation. Les filles racontent que depuis leur enfance leur vie a changé : 'Si tu veux faire des trucs dehors, tu restes pas dans la cité, tu vas ailleurs'. Sortir pour elles, c'est aller plus loin que le devant d'immeuble. 'On ne peut plus faire les choses comme avant. Les mecs, c'est pas grave, ça reste des mecs, mais nous, c'est mal vu'. Il est question de rester et de sortir, de demeurer, de devenir. 'Paris c'est la liberté, Champigny la sécurité'. Il est question d'une échappée nécessaire. Les travaux de Deleuze et Guattari trouvent un nouveau terrain d'application, nous aidant à considérer l'expérience minoritaire sous un angle optimiste, dès lors qu'elle incite à quitter et à chercher à s'accomplir ailleurs,

17 Citton, Y. 2010. L'avenir des humanités. Économie de la connaissance ou cultures de l'interprétation? Paris, La découverte.

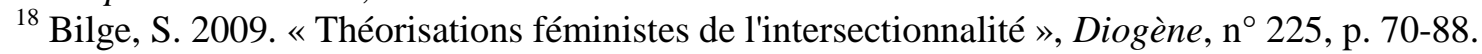

${ }^{19}$ Bouissou, C. 2010. « Chronique d'une sortie en ville», Revue artistique toulousaine Les Cahiers d'Adèle, numéro 5, « La ville ».

${ }^{20}$ Deleuze, G. et Guattari, F. 1980. Mille plateaux, Paris, Ed. Minuit. 
dans une altération du connu et du su. 'On peut pas faire partie de leur groupe comme avant, ça c'est sûr'. Ce dépaysement - 'Quand j'ai envie de prendre un peu l'air avec mes copines...' - est un dégagement des normes habituelles, des identifications ordinaires, des appartenances premières. Une chance peut-être pour une sortie du ban. Quitter le familier, s'exiler, se faire nomade. Une hypothèse se forme, encore à travailler : celle d'une souffrance plus vive chez ceux (les garçons) qui, s'ils se vivaient minoritaires, trouveraient des échappées. Ces filles expliquent qu'elles ne sont pas sous le joug du 'devoir être fort ', qu'elles se tiennent juste à côté, reconnaissent une situation asymétrique avec leurs pairs masculins, n'y laissent pas leur âme et se reconnaissent elles-mêmes et entre elles au travers d'un vécu commun et difficile mais non figé, d'une trajectoire polyvoque et nomade ${ }^{21}$.

Dans les pas de J. Borrell pour qui «la lumière ne luit qu'allumée, c'est-à-dire dans l'actualisation de sa puissance », nous entendons ces filles et leur intelligence au travail dans son énonciation, raison qui opère, «irrespectueuse mise en crise de l'ordre conceptuel » ${ }^{22}$ «Etre française c'est pas vivre à la française, je peux vivre comme je vis tous les jours, être naturelle et être française quand même. Ça veut dire quoi être française, ça veut dire quoi être française, ça veut dire quoi être française ?'. Provoquée par le sens commun elle y consent sans forcément y adhérer.

-Le reporter : 'On pourrait penser, vu de l'extérieur, que vous êtes communautaristes' ;

-Une fille : 'On pourrait oui, effectivement'

-Lui : 'Qu'est-ce que tu répondrais ?';

-Elle : 'J'ai rien à répondre, il suffit de me voir au quotidien et de voir que c'est pas ça'.

Travail de déprise, de dépouillement des mots impropres et de reprise. Travail de subjectivation qui réforme les rapports, les retourne, les transvalue. Travail de réforme de l'intelligence et de la raison. Mais de quelle raison est-il ici question?

Une raison immanente - détachée du Logos - passe par l'existence et l'exercice, opère par le mouvement, l'acte, le choix. Elle ne détruit pas le sensible, ne dissipe pas la résistance de l'autre en moi, ne nie pas son être autre. Elle est cette opération que tous les hommes ont en partage. Ni possession, ni ascèse, elle est ce qui transforme les relations entre les hommes. Son espace est espace du milieu, celui de la mesure. Elle est permise par la pensée du midi chère à $\mathrm{A}$. Camus ${ }^{23}$. Créative, elle provient des « risques que chacun est capable de prendre en mettant en question sa pensée, son langage, son temps et toute identité (sexuelle, nationale, ethnique, professionnelle, religieuse, philosophique...) qui s'y abrite $»^{24}$.

La raison est à ce niveau une lumière, une lumière propre à la condition humaine, allumée en soi-même. Produire en soi-même ce qui luit suppose que les choses et leur usage ne sont pas accordés d'avance et une fois pour toutes ; aucune harmonie n'est préétablie, mais une tension s'exerce, une mise en crise des opinions de la cité, qui restera précaire, toujours à refaire et toujours défaite ${ }^{25}$.

Les filles qui parlent entre elles et avec le journaliste au prénom arabe qui se meut aisément dans les problématiques de la vie dans la cité sont des Antigone, telles que les décrit J. Kristeva $^{26}$ : résistantes aux pouvoirs qui aliènent leurs vies, revendiquant une individualité

\footnotetext{
${ }^{21}$ Bouissou, C. 2011. Exil du banal et retour vers l'enfance. De l'autre côté de l'expérience. Colloque international Philosophie des frontières, Travaux et documents, PUV, $\mathrm{n}^{\circ} 51$.

${ }^{22}$ Borrell, J. 1993. La raison nomade, Paris, Payot, p. 224.

${ }^{23}$ Camus, A. 1951. L’homme révolté, Paris, Puf.

${ }^{24}$ Braconnier A. 2006. Entretien avec Julia Kristeva, Le Carnet PSY, n ${ }^{\circ} 110$, p. 40-47.

${ }^{25}$ Borrell, J. 1993. La raison nomade, Paris, Payot.

${ }^{26}$ Kristeva, J. 2008. Antigone, la limite et l'horizon, http://www.kristeva.fr/antigone.html.
} 
absolue pour elles-mêmes et pour leurs frères, ces garçons dont elles comprennent les raisons, sans s'y identifier ni y adhérer. «Epouse et n'épouse pas ta maison » dit R. Char ${ }^{27}$. Elles se savent plus fermement qu'eux engagées dans des projets d'existence - se marier, s'en sortir, côtoyer des gens différents tout en restant elles-mêmes -, tiennent au respect, de soi d'abord, et qu'il en soit de même pour l'autre, tiennent en respect ceux qui y verraient un signe d'oppression. France Culture se fait média de l'intelligence des raisons humaines, geste plus que propriété, raison qui se déprend d'elle-même en exerçant d'abord son irrespect sur ellemême, se déplaçant jusqu'à son bord ${ }^{28}$.

Enracinement incontesté, mobilité psychique, entendement des raisons de l'autre, voici ce dont témoigne cette polyphonie. Les filles construisent une santé normative au sens de Canguilhem ${ }^{29}$, permettant de se dégager des carcans dépréciateurs. Elles ne raisonnent pas abstraitement mais placent les sujets dans les paysages qui sont les leurs - forme de pensée typique du 'féminin ', altruiste et incarné - exprimant ainsi un rapport au collectif, s'autorisant à faire entendre leur voix dans la cité, au nom du droit naturel à la liberté, à l'égalité, à la dignité. Dans des éclats de rire, elles disent leur estime pour ceux qui les entourent. Elles rendent dignes leurs parents, les garçons qu'elles ne fréquentent plus comme avant, alter-egos auxquels elles sont fidèles.

Les filles se déterminent en prenant parole. Déconstruisent et s'instituent, ont des droits et des devoirs, font usage d'elles-mêmes. Travaillent. Aiment. Altèrent l'ordinaire et apprennent à se tenir dans une forme de solitude ${ }^{30}$. Dialoguant, elles mènent une opération de discernement, de séparation, sortent de la confusion, désamalgament. Concernées et solidaires, elles nomment les sans-noms, les hommes prolétaires, ceux qui n'ont droit ni aux ancêtres ni à l'expression publique: les hommes sans inscription symbolique, sans histoire majuscule ; mutiques, sans parole ni promesse, interdits. Elles se font l'écho des histoires et des souffrances des expériences in-ouïes, expropriées, spectrales tant qu'elles ne sont pas dites. Elles les sortent de leurs limbes, du séjour des enfants morts sans baptême, s'approprient les trajectoires de leurs pères.

Plasticité psychique et mobilité donnent au sujet sa force. C'est là que J. Kristeva ${ }^{31}$ identifie un génie féminin. Génie à entendre comme générateur, non pas don de soi au risque de s'y perdre, mais permanence du lien et de l'objet, souci de sauvegarder la pensée au nom de la vie, insistance sur le temps de l'éclosion et de la renaissance. La puissance, l'endurance et l'émancipation féminines passent par ce geste de réglage, de mesure des rapports : faire en soi une place pour l'autre, l'étranger en soi-même ${ }^{32}$. Trouver la distance, la juste place parmi et avec les autres. Le génie féminin n'est pas l'apanage des femmes, d'ailleurs la penseuse fait un pas supplémentaire et use d'un autre concept, celui de bisexualité psychique : possibilité de se prolonger en l'autre, d'être dans un rapport avec l'autre de telle manière que je passe dans l'autre sans le détruire, que je vais le chercher là où il, elle, est, sans essayer de tout ramener à moi. «Je est un autre » disait Rimbaud. Le monde n'est pas coupé en deux.

A notre tour d'entendre dans cette polyphonie radiophonique une puissance et un génie féminins : par le dialogue, les questions renvoyées au questionneur, les mots inappropriés refusés, les sauts opérés par-dessus les obstacles que sont les assignations non choisies, le lien gai, varié, nombreux, autorisant à parler de soi à plusieurs voix, font bouger les cadres

\footnotetext{
${ }^{27}$ Char, R. 1983. CEuvres Complètes. Les Feuillets d'Hypnos (Fragment 34), Paris, Gallimard, p.183.

${ }^{28}$ Borrell, J. 1993. La raison nomade, Paris, Payot.

${ }^{29}$ Canguilhem, G. 1947. Milieu et normes de l'homme au travail, Cahiers Internationaux de sociologie, vol. III, pp.120-136.

${ }^{30}$ Telle que la décrit J.-M. Quinodoz dans son ouvrage La solitude apprivoisée (2010, Paris, Puf).

${ }^{31}$ Kristeva, J. 2002. Le génie féminin. Colette ou la chair du monde (tome III), Paris, Fayard.

${ }^{32}$ Kristeva, J. 1988. Étrangers à nous-mêmes, Paris, Folio.
} 
habituels, les limites, les frontières. Les filles traversent les espaces citadins, interlocutoires, subjectifs, font advenir autre chose que l'arrêt sur image, le refuge dans le refus, la forteresse assiégée, défensive, crispée. Elles établissent un autre rapport, un autre gouvernement, de soi d'abord.

\section{Conclusion}

Réception d'un documentaire radiophonique, écoute flottante, lecture de textes multiples, là s'inscrit notre démarche, privilégiant le travail avec la profondeur des psychismes où les lois et l'ordre social sont susceptibles de muer, avant d'être reconnus et consacrés par la justice politique. Pour ce, le génie féminin est une voie/voix à générer. La confrontation aux épreuves, dans le sens le plus plein - cohabitation avec la pulsion de mort, considération de la négativité de toute expérience humaine - donne sérénité, force et solidité à ceux, à celles, qui en produisent une connaissance, un art ou un mode de vie/survie. Une pédagogie de l'altérité inscrite dans cette perspective dispose d'atouts véritables, cherchant des voies, esquissant des pistes pour les générations à venir qui en retrouveront les traces et s'y engageront. Plutôt que d'exclure la négativité humaine, il faut au contraire la travailler en organisant le pessimisme. Parce que l'émancipation est solidarité. 